\title{
$\beta$-Catenin-Gli1 interaction regulates proliferation and tumor growth in medulloblastoma
}

\author{
Jenny Zinke ${ }^{1}$, Fabian T Schneider ${ }^{2,4}$, Patrick N Harter ${ }^{1,3}$, Sonja Thom, Nicole Ziegler ${ }^{1}$, Rune Toftgård²,
} Karl H Plate ${ }^{1}$ and Stefan Liebner ${ }^{1 *}$

\begin{abstract}
Background: The Wnt/beta-catenin and the Hedgehog $(\mathrm{Hh})$ pathway interact in various cell types while eliciting opposing or synergistic cellular effects. Both pathways are known as exclusive drivers of two distinct molecular subtypes of medulloblastoma (MB).

In sonic hedgehog (Shh)-driven MB, activation of Wnt signaling has been shown to suppress tumor growth by either beta-catenin-dependent or -independent inhibition of Shh signaling. However, mechanistic insight in how beta-catenin inhibits the Hh pathway is not known.
\end{abstract}

Findings: Here we show that beta-catenin stabilization by the glycogen synthase kinase 3 inhibitor lithium chloride (LiCl) reduced growth of primary hedgehog-driven MB tumor spheres from patched heterozygous mice (Ptch ${ }^{+/}$) in vitro. LiCl treatment of $\mathrm{MB}$ spheres down-regulated the Hh target Gli1, whereas the repressive Gli3 protein (Gli3R) was increased. Mechanistically, we show by co-immunoprecipitation and proximity ligation assay that stabilized beta-catenin physically interacts with Gli1, leading to Gli1 sequestration and inhibition of its transcriptional activity. Reduction of Hh signaling upon $\mathrm{LiCl}$ stimulation resulted in reduced proliferation, sphere self renewal, a G2/M arrest and induction of a senescent-like state, indicated by p21 upregulation and by increased staining of senescence-associated beta-galactosidase (SA-betaGal). Moreover, LiCl treatment of subcutaneously transplanted MB cells significantly reduced tumor initiation defined as "tumor take". Although tumor progression was similar, $\mathrm{LiCl}$-treated tumors showed decreased mitotic figures and phospho-histone $\mathrm{H} 3$ staining.

Conclusion: We propose that beta-catenin stabilization increases its physical interaction with Gli1, leading to Gli1 degradation and inhibition of Hh signaling, thereby promoting tumor cell senescence and suppression of "tumor take" in mice.

Keywords: $\beta$-catenin, Gli1, Interaction, Medulloblastoma, Senescence, p21, LiCl, GSK-3 $\beta$

\section{Introduction}

In medulloblastoma (MB), the most common malignant pediatric brain tumor, Wnt pathway-driven tumors represent one of four distinct molecular subgroups with particularly favorable prognosis [1-4]. MB of the sonic hedgehog (Shh) subgroup account for up to $25-30 \%$ of human MBs and carry frequently mutations in the Shh receptor patched 1 (ptch1) [2,5-7]. The prognosis of Hh-driven MBs is less favorable then the one of Wnt-driven $\mathrm{MB}$ and despite

\footnotetext{
* Correspondence: stefan.liebner@kgu.de

'Institute of Neurology (Edinger-Institute), Johann Wolfgang

Goethe-University Frankfurt, Medical School, Heinrich-Hoffmann-Straße 7,

60528 Frankfurt, Germany

Full list of author information is available at the end of the article
}

current multimodality treatment, MB patients suffer from considerable treatment-induced side effects $[8,9]$.

In the "canonical" Wnt pathway $\beta$-catenin acts as a transcription factor with members of the lymphoid enhancer factor (Lef)/T-cell factor family [10]. On Wnt ligand-mediated activation of a complex formed by frizzled receptors and low-density lipoprotein receptor-related protein $5 / 6$, proteasomal degradation of $\beta$-catenin is inhibited by inactivating the destruction complex formed by glycogen synthase kinase $3 \beta$ (GSK-3 $\beta$ ), adenomatous poliposis coli (APC), and axin. Hedgehog, and specifically Shh, functions as a mitogen driving proliferation of granule neuron precursors in the cerebellum [11]. In the absence of Shh, patched (Ptch), a 12-transmembrane spanning protein, represses smoothened (SMO) thereby 
a

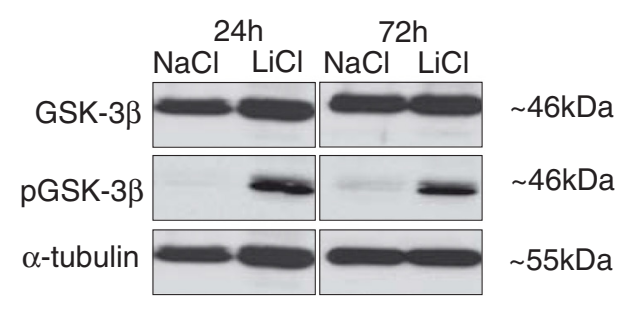

C

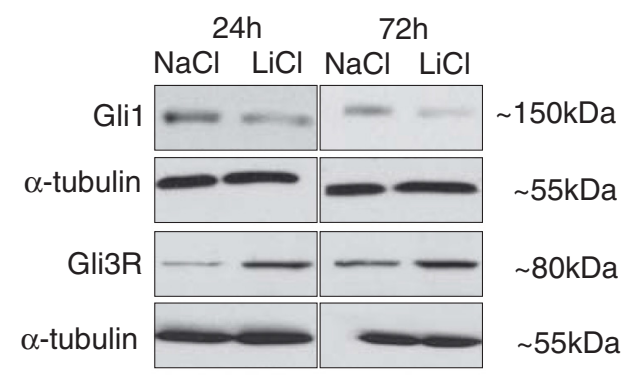

b

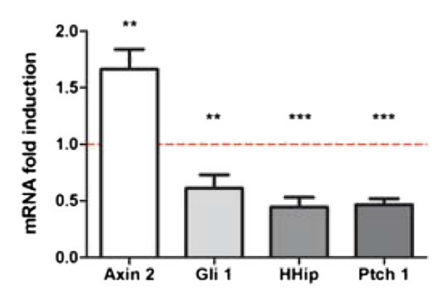

d

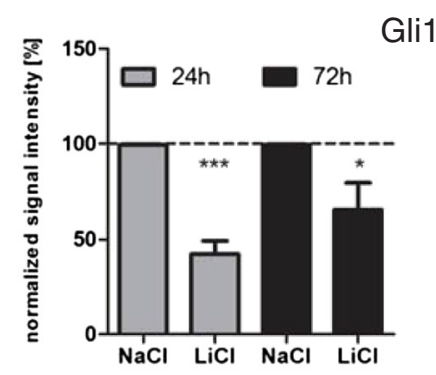

e

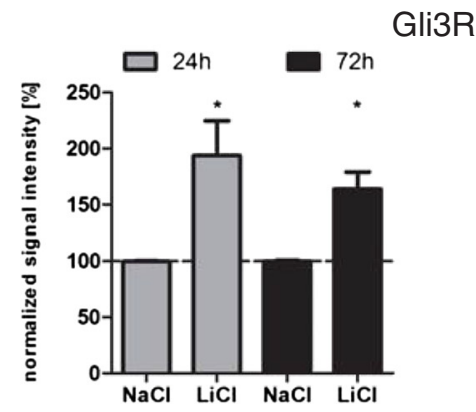

Figure 1 Activation of Wnt/ $\beta$-catenin signaling led to down-regulation of $\mathrm{Hh}$ target genes in primary $\mathrm{Ptch}^{+/-} \mathrm{MB}$ cells. (a) Primary Ptch ${ }^{+/}$ $\mathrm{MB}$ spheres treated for $24 \mathrm{~h}$ or $72 \mathrm{~h}$ with $10 \mathrm{mM} \mathrm{NaCl}$ or LiCl showed phosphorylated GSK-3 $\beta$ after treatment with LiCl. Whole cell lysates were

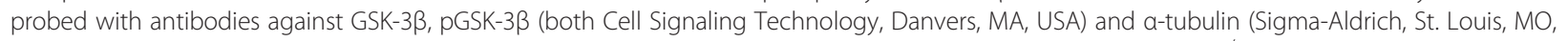
USA) as a loading control. (b) Expression of Axin2, Gli1, Ptch1 and Hhip mRNA was evaluated in primary Ptch ${ }^{+/-}$MB tumor cells after $72 \mathrm{~h}$ treatment with $10 \mathrm{mM} \mathrm{NaCl}$ or LiCl. Bars represent mean \pm s.d. ( $n=7$, p-values left to right: $\left.{ }^{* *} 0.0064,{ }^{* 0} 0.0186,{ }^{* * *} 0.0001,{ }^{* * *} 0.0002\right)$. Expression of Hh target genes was down-regulated by LiCl-treatment. (c) Gli1 protein levels decreased and Gli3R protein levels increased after LiCl treatment. Membranes were probed with antibodies against Gli1 (R\&D Systems, Minneapolis, MN, USA) and a-tubulin (Sigma-Aldrich). (d) Quantification of signal intensity for Gli1 normalized to a-tubulin (ImageJ 1.47v software). Bars represent signal intensity after $24 \mathrm{~h}$ (grey) and $72 \mathrm{~h}$ (black) of $\mathrm{NaCl}$ and LiCl treatment. ( $n=5$, p-values left to right: *** $<0.0001,{ }^{*} 0.0383$ ). Gli1 levels decreased after treatment with LiCl. (e) Quantification of signal intensity for Gli3R normalized to a-tubulin (ImageJ 1.47v software). Bars represent signal intensity after $24 \mathrm{~h}$ (grey) and $72 \mathrm{~h}$ (black) of NaCl and LiCl treatment. ( $n=3, \mathrm{p}$-values left to right: * $\left.0.0401,{ }^{*} 0.0137\right)$. Gli3R levels increased after treatment with LiCl. (Analysis done in GraphPad Prism 5.01 software).

inhibiting Hh signaling. SMO is a member of the 7-transmembrane spanning G-protein-coupled receptor-like superfamily. On Shh binding to Ptch its repressive function on SMO is released, thereby activating Gli1/2 dependent transcription. In the repressive state Gli1/2 proteins are phosphorylated, ubiquitinated and degraded [12].

Interestingly, Wnt and Shh were shown to interact in various cell types and organs during development and in the adult, while eliciting opposing or synergistic cellular effects. Generally, it has previously been shown that Shh inhibits the Wnt pathway during tongue papilla development and in squamous cell carcinoma $[1,3,4,13,14]$.

Recently, also in MB interaction of the Wnt and $\mathrm{Hh}$ pathway has been shown, describing either a $\beta$-catenindependent or -independent inhibition of Shh signaling by Wnt $[5,6,15]$.

However, neither mechanistic insight into $\beta$-cateninmediated inhibition of the Hh pathway, nor the therapeutic potential of $W n t / \beta$-catenin-activating drugs has been examined specifically in $\mathrm{MB}$. 
Table 1 List of qRT-PCR primers

\begin{tabular}{ll}
\hline Primername & Sequence \\
\hline Axin 2_s & GCCGACCTCAAGTGCAAACTC \\
Axin 2_as & GGCTGGTGCAAAGACATAGCC \\
Gli 1_s & CCTTAGCAATGCCAGTGACC \\
Gli 1_as & GAGCGAGCTGGGATCTGTGTAG \\
Hhip_s & GGCCTCACGACCACATTCTTC \\
Hhip_as & AGCCATCAGGACCAAAGAGCA \\
Ptch1_s & TTGGGATCAAGCTGAGTGCTG \\
Ptch1_as & CGAGCATAGCCCTGTGGTTCT \\
P21_s & CTGGAGGGCAACTTCGTCTGG \\
P21_as & GAGTGCAAGACAGCGACAAGG \\
\hline
\end{tabular}

Here we show that the FDA-approved drug $\mathrm{LiCl}$, which results in $\beta$-catenin stabilization via GSK-3 inhibition, suppressed formation of $\mathrm{Ptch}^{+/-} \mathrm{MB}$ tumor spheres as well as tumor take in mice. We provide mechanistic evidence that this effect is dependent on the physical interaction of Gli1 and $\beta$-catenin, leading to Gli1 degradation, G2/M cell cycle arrest and cellular senescence.

\section{Findings}

Primary MB cells isolated from $\mathrm{Ptch}^{+/-}$mice [8] were treated with $10 \mathrm{mM} \mathrm{LiCl}$ in vitro. Inhibition of GSK-3 by LiCl, evidenced by GSK-3 phosphorylation (Figure 1a), led to reduced tumor sphere self-renewal (Additional file 1: Figure S1a) as well as to reduced tumor cell growth (Additional file 1: Figure S1b). In accordance, $\beta$-catenin stabilization and transcriptional activation, confirmed by increased Axin2 mRNA (Figure 1b) and protein levels (Additional file 2: Figure S2), led to the downregulation of the Hh targets Gli1, Ptch1 and Hhip, on the mRNA level evidenced by qRT-PCR (see Table 1 for primer list), suggesting an inhibitory effect of $\beta$-catenin on Hh-induced transcription (Figure 1b). The decrease of Gli1 as well as an increase in $\beta$-catenin protein stability was verified by treatment with (2'Z,3'E)-6-Bromoindirubin-3'-oxime (6BIO), a non-FDA-approved but highly specific inhibitor of GSK-3 (Additional file 3: Figure S3) [16].

As shown in the developing spinal cord, activated Wnt/ $\beta$-catenin signaling increases the expression of repressive Gli3 (Gli3R), which in turn inhibits the $\mathrm{Hh}$ pathway $[4,11]$. We corroborated Gli3R up-regulation upon $\mathrm{LiCl}$ treatment in $\mathrm{Ptch}^{+/-} \mathrm{MB}$ spheres on the protein level (Figures 1c, d, e). However, we cannot judge on the direct regulation of Gli3R via $\beta$-catenin. It should be noted that a ternary complex between Gli3R/ $\alpha$-catenin/ $\beta$-catenin has been observed in chondrocytes, leading to the inhibition of $\beta$-catenin transcriptional activity $[2,7,17]$. In the $\mathrm{Ptch}^{+/-}$ $\mathrm{MB}$ tumor spheres, we were not able to detect such a complex by co-immunoprecipitation at $8 \mathrm{~h}$ of $\mathrm{LiCl}$ treatment (data not shown).

Although the up-regulation of Gli3R and the concomitant down-regulation of Ptch1, Hhip and Gli1 either on mRNA or protein level fit to the interpretation of Hh pathway inhibition by $\mathrm{LiCl}$-mediated $\beta$-catenin stabilization, the mechanism of Gli1 down-regulation remained elusive. To evaluate if proteasomal degradation diminishes Gli1 we investigated the effect on Gli1 protein by the proteasome inhibitor MG-132. LiCl-mediated decrease of Gli1 was partly reversed by proteasomal inhibition (Figures 2a, b). However, the exact mechanism of Gli1 degradation upon $\beta$-catenin stabilization requires further investigation.

Furthermore, $\mathrm{LiCl}$ treatment significantly decreased cell proliferation, indicated by diminished Ki67-positive cells (Figure 3a) and by decreased cells marked by the more specific mitotic marker phospho-histone H3 (pHH3; Figure $3 \mathrm{~b}$ ). Hence, $\mathrm{LiCl}$ treatment led to an accumulation

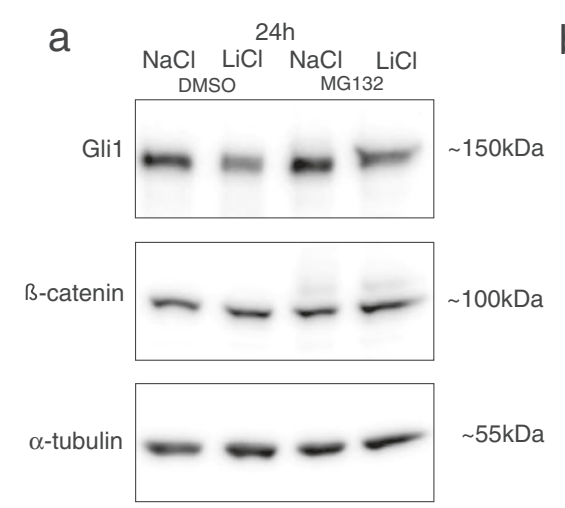

b

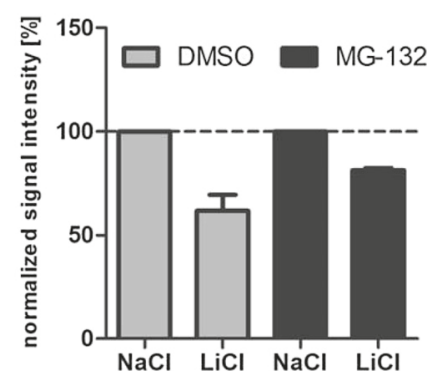

Figure 2 Gli1 proteasomal degradation is partly reduced with MG-132. (a) Ptch ${ }^{+/-} \mathrm{MB}$ cells were treated with $10 \mathrm{mM} \mathrm{NaCl}$ or $\mathrm{LiCl}$ for $24 \mathrm{~h}$. $10 \mu \mathrm{M}$ of proteasome inhibitor MG-132 (A. G. Scientific) or DMSO was added $4.5 \mathrm{~h}$ before lysing cells for Western blot analysis. Membranes were probed against Gli1 (R\&D), $\beta$-catenin (BD Transduction Laboratories, San Jose, CA, USA) and a-tubulin (Sigma-Aldrich). (b) Bars represent mean \pm s.d. for Gli1 level after $24 \mathrm{~h}$ of $\mathrm{NaCl}$ or $\mathrm{LiCl}$ treatment and $4.5 \mathrm{~h}$ treatment with DMSO (grey) or MG-132 (black). The decrease of Gli1 protein levels under $\mathrm{LiCl}$ treatment was partly reversed by proteasomal inhibition ( $n=2$, Analysis done in GraphPad Prism 5.01 software). 


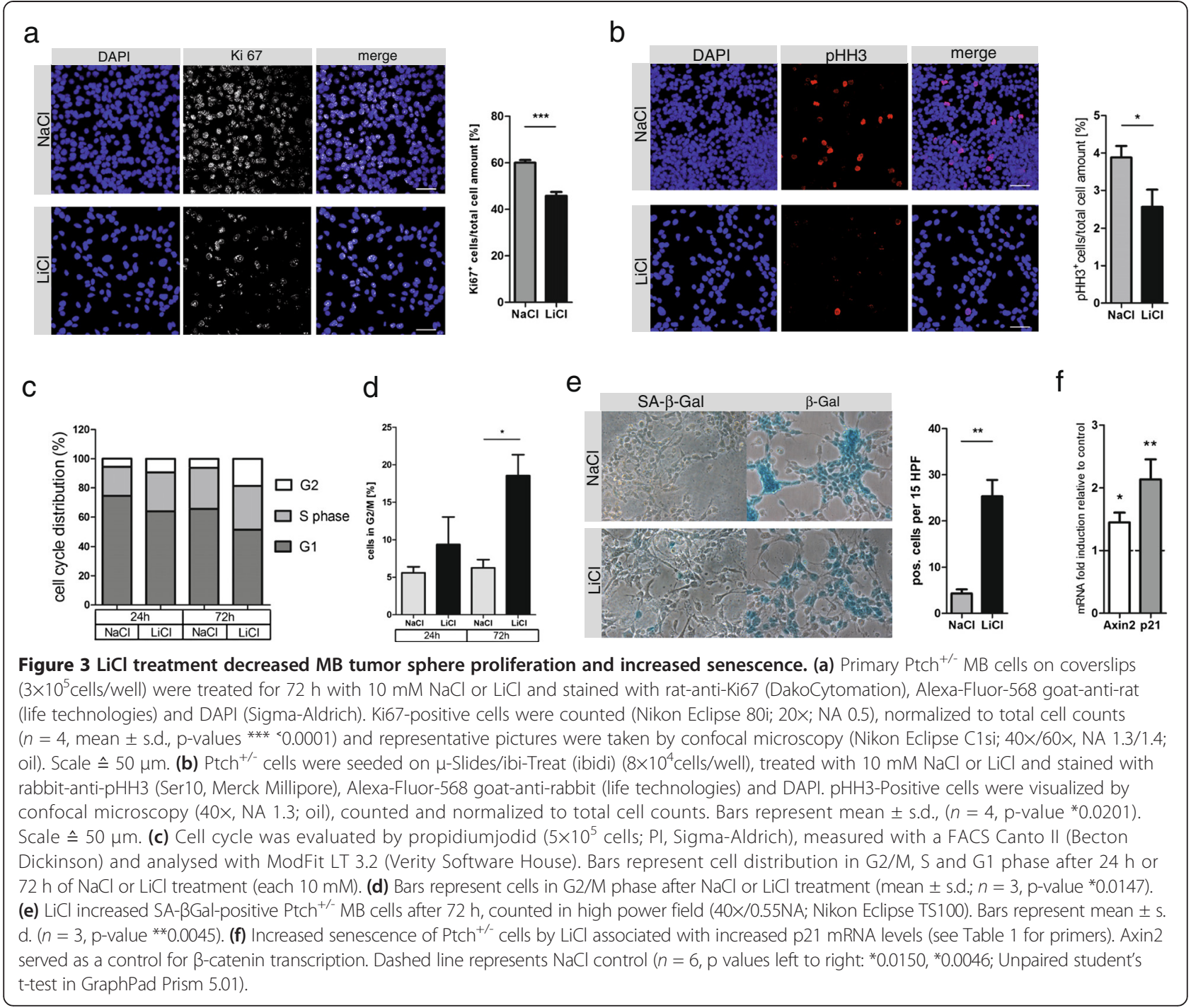

of cells in G2/M cell cycle phase (Figures 3c, d). As shown recently, G2/M accumulation also occurs in LiCl-treated endometrial and glioma cancer cell lines [13,14], and $\mathrm{LiCl}$ treatment of pancreatic ductal adenocarcinomas reduces the tumorigenicity of cells through $\mathrm{Hh}$ inhibition [15].

Along this line, here we show that in primary $\mathrm{Ptch}^{+/-}$ $\mathrm{MB}$ cells $\mathrm{LiCl}$ treatment increased the number of senescence-associated $\beta$-galactosidase (SA- $\beta$ Gal)-positive cells (Figure 3e) and upregulated p21 after $72 \mathrm{~h}$ as a senescence marker (Figure 3f). These findings suggest that $\mathrm{LiCl}$ induces a senescent-like state in $\mathrm{MB}$ tumor cells. Apoptosis however, was not detectable after $8 \mathrm{~h}, 24 \mathrm{~h}$ and $72 \mathrm{~h}$ of $\mathrm{LiCl}$ treatment investigated by annexin $\mathrm{V}$ staining (data not shown). Furthermore, $\mathrm{LiCl}$ treatment did not specifically increase autophagy in $\mathrm{Ptch}^{+/} \mathrm{MB}$ spheres, evidenced by staining for LC3 (data not shown). However this aspect requires further investigation.
We next analyzed the downstream effects of GSK3 inhibition with respect to $\mathrm{Wnt} / \mathrm{\beta}$-catenin and Hh crosstalk, as $\beta$-catenin interaction with Gli1 has previously been suggested to occur in endometrial carcinoma in which both factors cooperate to drive tumor growth [18]. To this end we immunoprecipitated $\beta$-catenin from $\mathrm{Ptch}^{+/-} \mathrm{MB}$ cultures after $8 \mathrm{~h} \mathrm{LiCl}$ stimulation and blotted for Gli1, which was significantly increased upon $\beta$-catenin stabilization by $\mathrm{LiCl}$, although low amounts of co-precipitation was also detectable in the $\mathrm{NaCl}$ controls (Figure 4a). This suggests that stabilized $\beta$-catenin is able to sequester Gli1, making Gli1 unavailable for transcriptional activity and likely subjecting it to protein degradation.

To visualize the $\beta$-catenin-Gli1 interaction upon $\mathrm{LiCl}$ stimulation, we performed a proximity ligation assay (PLA) with antibodies against $\beta$-catenin and Gli1. Indeed, this approach corroborated the $\beta$-catenin-Gli1 


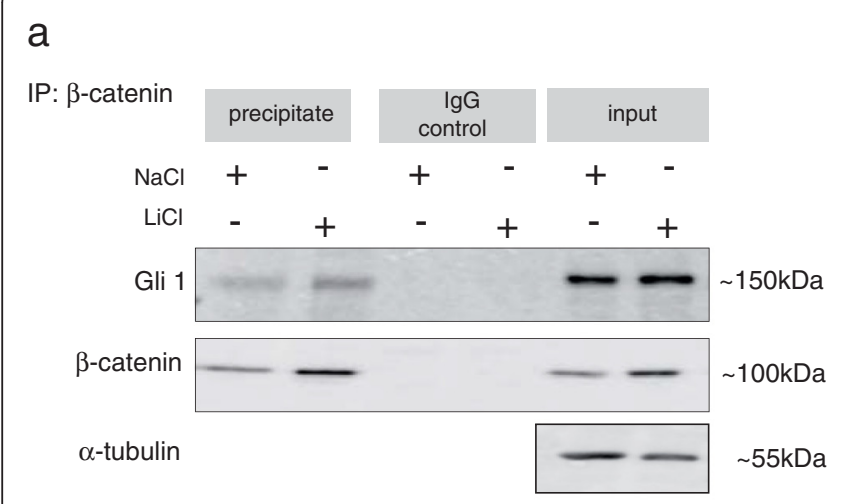

b
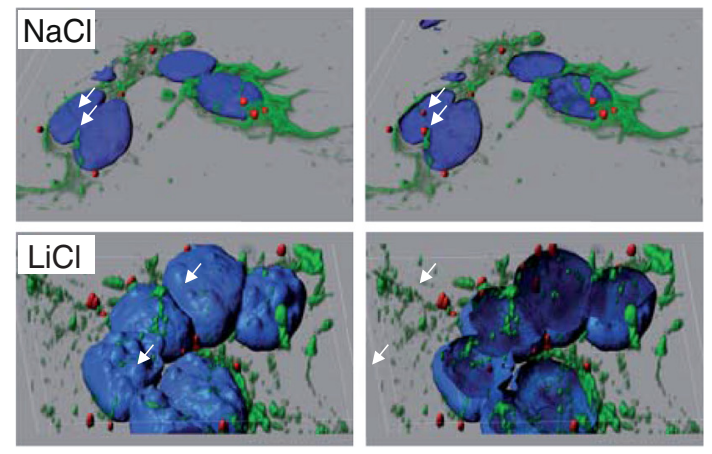

C

d
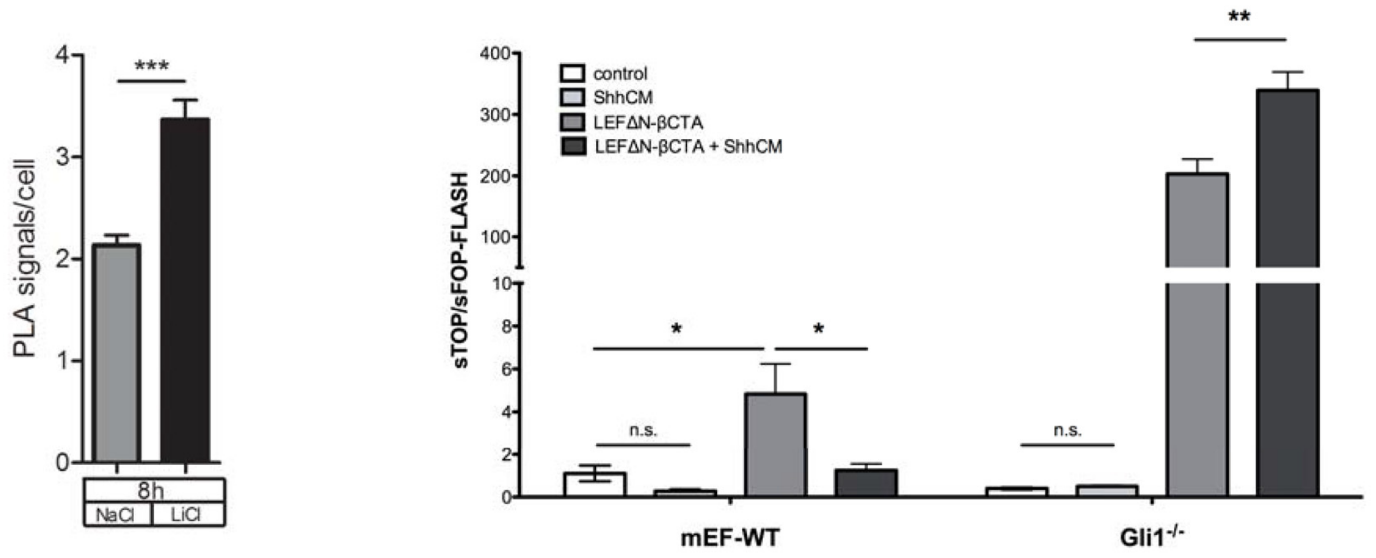

Figure $4 \mathrm{Gli1}$ interacts with $\beta$-catenin in $\mathrm{Ptch}^{+/-} \mathrm{MB}$ tumor spheres. (a) Primary $\mathrm{Ptch}^{+/-} \mathrm{MB}$ cells were treated with $\mathrm{NaCl}$ or $\mathrm{LiCl}(8 \mathrm{~h})$ and the extracts were immunoprecipitated with an anti- $\beta$-catenin antibody (BD Transduction Laboratories) prebound to protein G-agarose (Roche, Basel, Switzerland). Co-precipitated Gli1 (R\&D Systems) was verified by Western blotting. (b) For PLA, primary Ptch ${ }^{+/}$MB cells were seeded on $\mu$-slides (ibidi), treated with $\mathrm{NaCl}$ or $\mathrm{LiCl}$ for $8 \mathrm{~h}$, incubated with anti-Gli1 (R\&D Systems) and anti- $\beta$-catenin antibodies (BD Transduction Laboratories), corresponding anti-goat (minus) or anti-mouse (plus) PLA probes and ligation-mix was added (Duolink In Situ, Detection Kit orange; Excitation: 554 nm, Emission: 579 nm, Sigma-Aldrich). Samples were examined by confocal microscopy (Nikon Eclipse C1si; 40x/60x, NA 1.3/1.4; oil; 50pictures/condition). Amaris (7.7; Bitplane, Switzerland) was used to generate surface-rendered nuclei from 60x images; a clipping plane was introduced to visualize nuclear PLA signals. Co-immunoprecipitation and PLA revealed an interaction between $\beta$-catenin and Gli1, which increases under LiCl treatment. (c) Total number of interactions (red dots; protein-protein distance $\leq 40 \mathrm{~nm}$ ) was normalized to the total number of DAPI-positive nuclei per image. Bars represent PLA signals/cell after $8 \mathrm{~h}$ treatment with $\mathrm{NaCl}$ (grey) and LiCl (black). Analysis was done with GraphPad Prism 5.01 software (p-value 0.0004). (d) $5 \times 10^{4} \mathrm{mEF-WT}$ and $\mathrm{mEF-Gli1}{ }^{--}$cells were seeded per $24-$ well and transfected after $24 \mathrm{~h}$ with either $8 \times$ SuperTOP or $8 \times$ SuperFOP (380 ng), TK-Renilla (20 ng) and LEF $\Delta$ N-BCTA (100 ng) or empty vector pcDNA3.1 (100 ng) using $4 \mu$ l Lipofectamine LTX with $0.5 \mu$ l PLUS reagent (Life Technology) in Optimem (Life Technology). $24 \mathrm{~h}$ post transfection cells were treated with Shh-conditioned medium (ShhCM) for $24 \mathrm{~h}$. Luciferase activity was measured on a TECAN plate reader using the Dual Luciferase Kit (Promega). $(n=5)$, p-values left to right: ${ }^{*} 0.0331,{ }^{*} 0.0372,{ }^{* *} 0.0075$.

interaction observed in co-precipitation experiments (Figures 4b, c, Additional file 4: Figure S4). Furthermore, 3D reconstruction of confocal Z-stacks revealed the localization of a small fraction of $\beta$-catenin-Gli1 within the nucleus, whereas most of the complex localized to the cytoplasm of $\mathrm{Ptch}^{+/} \mathrm{MB}$ tumor cells (Figure 4b). This observation suggests that $\beta$-catenin might sequester Gli1 in the nucleus as well as in the cytoplasm, thus inhibiting Gli1-mediated transcription. As published previously in tongue taste papilla turnover, in squamous cell carcinoma and in gastric cancer, the Hh pathway can equally inhibit $\beta$-catenin transcriptional activity $[4,19]$.

Taking advantage of the putative mutual inhibition of Gli1 and $\beta$-catenin and to further analyze the specific role of Gli1 for functional interaction with $\beta$-catenin, we explored the inhibitory function of Shh on $\beta$-catenin signaling using WT as well as $\mathrm{Gli1}^{-1-}$ mouse embryonic fibroblasts (MEFs). MEFs were transfected with the chimeric construct LEF $\triangle \mathrm{N}-\beta C \mathrm{CTA}$, conferring dominant $\beta$-cateninLEF transcription, and subsequently stimulated with control 
or Shh-conditioned medium (ShhCM) [20]. As a read-out we utilized the superTOP/FOP-FLASH assay, measuring luciferase activity driven by a LEF-responsive promotor [21]. Interestingly, the inhibitory function of ShhCM on $\beta$-catenin transcription was completely abrogated in $\mathrm{Gli1}^{-1-}$ MEFs (Figure 4d), suggesting that the interaction of Gli1 with LEF $\triangle N-\beta C T A$ is required for transcriptional inhibition. As the LEF $\triangle \mathrm{N}-\beta C \mathrm{CTA}$ construct contains only the C-terminal transactivation domain of $\beta$-catenin (amino acid 695-781), these findings are suggestive that the $C$-terminus of $\beta$-catenin is crucial for the interaction with Gli1. It will be interesting to analyze in detail which exact binding-domains of $\beta$-catenin and Gli1 are required for protein-protein interaction.

Furthermore, we investigated the role of $\beta$-catenin for hedgehog-mediated MB sphere growth by knocking down $\beta$-catenin by a siRNA approach. Interestingly, knock-down of $\beta$-catenin abrogated the growth inhibitory function of $\mathrm{LiCl}$ treatment (Additional file 5: Figure S5). Together these findings suggest that $\beta$-catenin stabilization and availability is crucial for $\mathrm{LiCl}$-induced Hh pathway inhibition via the sequestration of Gli1.

Finally, in order to understand if $\beta$-catenin activation might be beneficial for MB patients, we transplanted primary mouse $\mathrm{Ptch}^{+/}$MB cells subcutaneously into the flanks of $\mathrm{NMRI} /$ nude mice and treated the animals with either $\mathrm{LiCl}$ (7,5 $\mu \mathrm{l} / \mathrm{g}$ body weight, $1.2 \mathrm{M})$ or $\mathrm{NaCl}(7,5 \mu \mathrm{l} / \mathrm{g}$ body weight, $1.2 \mathrm{M})$ as control $[9,22]$.

$\mathrm{LiCl}$ treatment resulted in a significant delay in initial tumor incidence that we defined as "tumor take", ranging between day 22 and 26 (Figure 5b). Nevertheless, nearly all animals, irrespective of the treatment, developed tumors by day 32, with a tendency of smaller tumors in the $\mathrm{LiCl}$ group (Figures 5a, b). Taking together this finding suggests that $\mathrm{LiCl}$ treatment significantly inhibits tumor take (Figure $5 b$ ), although we did not observe a significantly reduced tumor burden at the experimental endpoint.

However, we observed reduced mitotic figures in LiCl-treated tumors, which was supported by decreased phospho-histone H3 (pHH3)-positive cells (Figures 5c, d). a
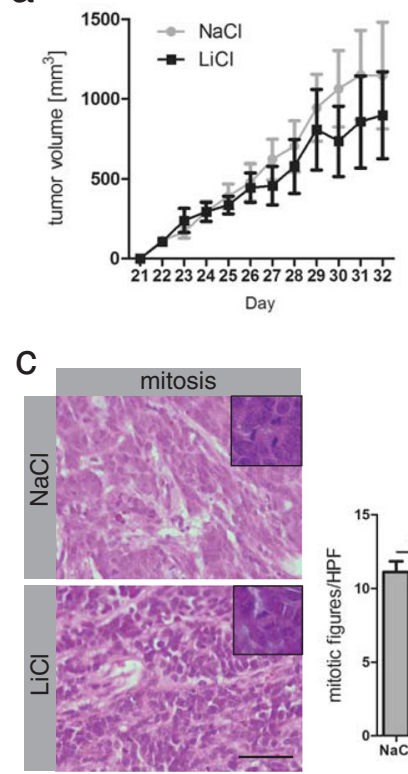

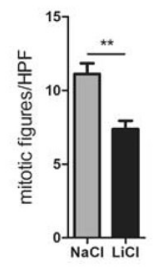

b

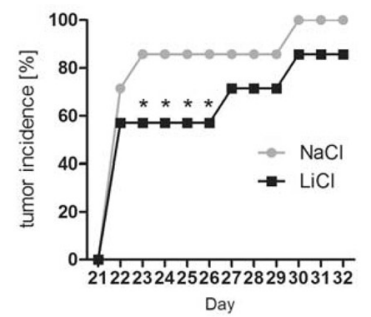

d

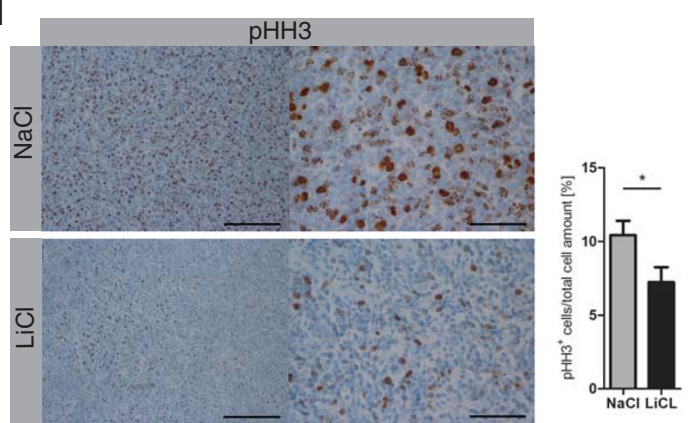

Figure $\mathbf{5} \mathrm{LiCl}$ treatment delayed growth and decreased volume of $\mathrm{Ptch}^{+/-}$MB-derived tumors. (a) $1 \times 10^{6}$ cells in $100 \mu$ l PBS were subcutaneously

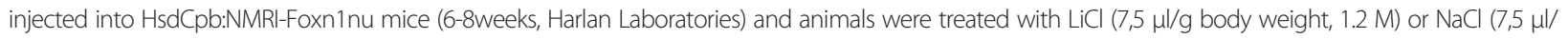
$\mathrm{g}$ body weight, $1.2 \mathrm{M}$ ) from day 5 post-transplantation. From day 21 tumors were measured daily thereafter (electronic disk micrometers, Vogel Germany), the mean of tumor volume was determined by the formula: $\pi / 6 \times$ maximal-diameter $x$ minimal-diameter ${ }^{2}\left(\mathrm{~mm}^{3}\right)$ until animals were sacrificed by day 32 . (b) Dots represent the percentage of tumor-bearing animals. Statics were performed using PSPP 0.8 .2 as described previously [23]. Equal numbers ( $n=7$ ) from $\mathrm{NaCl}$ - or LiCl-treated mice were tested for tumor formation probability by a non-parametric one-tailed binomial proportions test, setting the expected outcome for tumor formation to 90\%. There was no significant difference between the expectation and the tumor incidence for the NaCl-treated group (p-value 0.52), but for the LiCl-treated group (p-value ${ }^{*} 0.03$ ). (c) Paraffin sections $(3 \mu \mathrm{m})$ of tumors were stained with hematoxylin and eosin, mitotic figures were counted per high power field and pictures were taken at an Olympus BX50 microscope 40x (NA 0.75). Bars represent mean \pm s.d. $(n=7$ (NaCl), $n=6$ (LiCl)), p-value ${ }^{* *} 0.0021$ ). Scale bar $\triangleq 50 \mu \mathrm{m}$. Analysis was done with GraphPad Prism 5.01. (d) Immunohistochemistry with anti-pHH3 (Merck Millipore) antibody on paraffin sections ( 3 um) was performed using Ventana Discovery XT system (Ventana, USA), examined with the Axiophot (Zeiss, Germany, Achroplan 0.65) and analyzed in Stereo Investigator 4.34 (MicroBright Field.Inc Europe, Germany). Pictures were taken at a Nikon Eclipse 80i (Nikon, Japan; 10x/40x; NA 0.5). Bars represent mean \pm s.d. $(n=7$ (NaCl), $n=6$ (LiCl), p-value *0.0463). Scale bar represent $200 \mu m$ (left), $50 \mu m$ (right). 
This reflects a reduced population of cells in $M$ phase and suggests that the majority of cells are arrested in G2 phase, corroborating our in vitro findings (Figure 3b). Apoptosis was not altered in $\mathrm{LiCl}$-treated $\mathrm{MB}$ tumors, evidenced by cleaved caspase 3 staining (Additional file 6: Figures S6 a, b).

It is interesting however, that $\mathrm{LiCl}$ tumors showed decreased necrotic areas (data not shown), although tumor size did not significantly differ from controls. In this regard it is worth mentioning that we recently reported on a normalizing effect of $\beta$-catenin activation in endothelial cells of glioma vasculature [24]. This would support the hypothesis that $\mathrm{LiCl}$ treatment of subcutaneous $\mathrm{MB}$ tumors might affect both, cell proliferation as well as tumor microenvironment by normalizing the vasculature. When we analyzed $\mathrm{MB}$ tumors for vessel density and smooth muscle cell coverage by CD31 and $\alpha$ SMA staining, respectively, we did not observe significant differences between the $\mathrm{NaCl}$ - and the LiCl-treated group (Additional file 6: Figure S6 c, d). This result might be explained by the different tumor models used in the study of Reis et al. and the present manuscript. Glioblastoma are well known to be highly angiogenic, whereas $\mathrm{MB}$ does not strongly induce vessel growth [25]. This requires more in depth evaluation, also at different stages of tumor growth that is however beyond the scope of this study.

The overall tumor histopathology of the subcutaneous $\mathrm{Ptch}^{+/-}$tumors did not differ with respect to extracellular matrix and GFAP staining (Additional file 7: Figure S7).

The systemic treatment with $\mathrm{LiCl}$ and the resulting stabilization of $\beta$-catenin will always target more cell types then the tumor cell itself. Consequently, we have to estimate the benefit of the treatment by the net outcome of tumor burden. It should be noted that the clinical impact of these findings might relate to some types of Shh-driven MB that occur in infants and young adults, which are difficult to treat with established regimes or by Smo inhibitors that are currently in clinical trials [26]. It seems to be common to these treatment-resistant MBs that they carry mutations downstream of Ptch or Smo in genes such as suppressor of fused $(\mathrm{SuFu})$ or Gli2. Herein the stabilization of $\beta$-catenin at an early time point of the disease might have beneficial effects by directly targeting Gli1 and by supporting the chemotherapeutic treatment of the tumor cells. It should be noted that it is likely that human Shhdriven $\mathrm{MB}$, harboring a mutation in Ptch, also respond to $\beta$-catenin activation with reduction of Gli1. However, more work is required to evaluate the translational potential of our finding.

In summary our data show for the first time that activation of Wnt/B-catenin signaling by $\mathrm{LiCl}$ treatment inhibits proliferation of Shh-driven mouse MB via the interaction of $\beta$-catenin and Gli1.

This supports the concept that Gli1 interacts with $\beta$-catenin by default, and that an increase of stabilized $\beta$-catenin is able to sequester an increased amount of Gli1 and vice versa. This scheme of a balanced interaction implies that $\beta$-catenin stabilization could be titrated to a level at which virtually all Gli1 becomes sequestered, without affecting canonical Wnt pathway activation. These findings may open novel possibilities for therapeutic interventions for $\mathrm{MB}$ patients.

\section{Additional files}

Additional file 1: Figure S1. LiCl treatment reduced tumor cell selfrenewal and growth. (a) Ptch ${ }^{+/} \mathrm{MB}$ cells were cultivated for one week with either $10 \mathrm{mM} \mathrm{NaCl}$ or $\mathrm{LiCl}$, growth factors and $\mathrm{NaCl} / \mathrm{LiCl}$ were added every third day. After 7 days tumor spheres were counted, dissociated mechanically and subsequently seeded for a second and third sphere forming clonal assay (CA1 - 3). Ptch ${ }^{+-} \mathrm{MB}$ cells were kept on either $\mathrm{NaCl}$ or $\mathrm{LiCl}$ to investigate if $\mathrm{Ptch}^{+-} \mathrm{MB}$ cells might adapt to the LiCl treatment. p-Values left to right: ${ }^{* *} 0.0020,{ }^{* * *} 0.0006,{ }^{*} 0.0104$. Ptch ${ }^{+/}$MB cells seeded for three subsequent clonal assays (CA) showed no adaption to the LiCl treatment. (b) Primary $\mathrm{Ptch}^{+/} \mathrm{MB}$ cells were seeded on fibronectin/poly-L-ornithin-coated 96-well plates $\left(1 \times 10^{4} \mathrm{cells} /\right.$ well) and cultured overnight. Cells were treated with descending concentrations of $\mathrm{NaCl}$ or $\mathrm{LiCl}$ for $72 \mathrm{~h}$ and stained with crystal violet. Absorbance at $595 \mathrm{~nm}$ was measured (TECAN reader infinite M200 pro, TECAN, Männedorf, Switzerland) and crystal violet background staining was subtracted. Bars represent mean \pm s.d., $(n=4)$, p-values left to right: ** $0.0098, * 0.0478$.

Additional file 2: Figure S2. $\beta$-Catenin stabilization by LiCl treatment increased Axin2 protein level. (a) Primary Ptch ${ }^{+/} \mathrm{MB}$ spheres were treated with $10 \mathrm{mM} \mathrm{NaCl}$ or LiCl and harvested after $24 \mathrm{~h}$ and $72 \mathrm{~h}$. Membranes were probed with antibodies against Axin2 (Abcam) and a-tubulin (Sigma-Aldrich) as a loading control. Bars represent mean \pm s.d of Axin2 protein level after $24 \mathrm{~h}$ (grey) and $72 \mathrm{~h}$ (black) $\left(n=4, \mathrm{p}\right.$-value $\left.{ }^{*} 0.0164\right)$.

Additional file 3: Figure S3. $\beta$-Catenin stabilization by $6 \mathrm{BIO}$ treatment decreased Gli1 protein level and pHH3 positive cells. (a) Primary Ptch ${ }^{+\prime}$ $\mathrm{MB}$ spheres were treated with $10 \mu \mathrm{M} 6 \mathrm{BIO}$ or DMSO as control, harvested after $72 \mathrm{~h}$ and lysed and separated in cytoplasmic (C) and nuclear fraction (N). Membranes were probed with antibodies against Gli1 (R\&D Systems) and $\beta$-catenin (BD Transduction Laboratories). Lamin B1 (Abcam) and a-tubulin (Sigma-Aldrich) served as loading controls. Gli1 protein levels decreased, $\beta$-catenin protein levels increased under $6 \mathrm{BIO}$ treatment. (b) Primary Ptch ${ }^{+-} \mathrm{MB}$ cells were seeded on $\mu$-Slide 8 well ibi-Treat slides (ibidi, Martinsried, Germany) $\left(8 \times 10^{4}\right.$ cells/well) and treated with $10 \mu \mathrm{M}$ $6 \mathrm{BIO}$ or DMSO as control. Immunocytochemistry was performed with rabbit-anti-pHH3 (Ser10) antibody (Merck Millipore) followed by Alexa-Fluor56 goat-anti-rabbit antibody (life technologies) and DAPI. pHH3-positive cells were counted at a confocal microscope (Nikon Eclipse C1si; 40x, NA 1.3; oil immersion and normalized to total cell count. Bars represent mean \pm s.d. ( $n=3$, p-value $\left.{ }^{* * *}<0.0001\right)$. Scale bar represents $50 \mu \mathrm{m}$. The number of $\mathrm{pHH} 3$ positive cells decreased under treatment with 6BIO.

Additional file 4: Figure S4. Increased interaction of $\beta$-Catenin with Gli1 upon LiCl stimulation. For PLA, primary Ptch ${ }^{+/} \mathrm{MB}$ cells were seeded on $\mu$-slides (ibidi, Martinsried, Germany) and treated with $\mathrm{NaCl}$ or LiCl for $8 \mathrm{~h}$. Cells were incubated with antibodies against Gli1 (R\&D Systems) and $\beta$-catenin (BD Transduction Laboratories) and corresponding anti-goat (minus) or anti-mouse (plus) PLA probes. Ligation-mix, consisting of two oligonucleotides, and amplification-mix, consisting of nucleotides and fluorescently labeled oligonucleotides, was added (Duolink In Situ, Detection Kit orange (Excitation: 554 nm, Emission: 579 nm, Sigma-Aldrich). Samples were examined by confocal microscopy (Nikon Eclipse C1si; 40X/ 60x, NA 1.3/1.4; oil immersion; 50 pictures/condition).

Additional file 5: Figure S5. $\beta$-catenin knock down with siRNA abrogated the growth inhibitory function of $\mathrm{LiCl}$ treatment. Primary $\mathrm{Ptch}^{+/}$cells were transfected with either $5 \mathrm{nM}$ or $10 \mathrm{nM}$ of Silencer ${ }^{\circledR}$ Select Pre-designed siRNA against $\beta$-catenin or a control\#1 siRNA (Ambion, life technologies), MetafectenePro was used as transfection reagent and incubated for $5 \mathrm{~h}$. (a) $24 \mathrm{~h}$ post transfection cells ( $5 \mathrm{nM}$ and $10 \mathrm{nM}$ ) were treated with either $\mathrm{NaCl}$ 
or LiCl for $72 \mathrm{~h} .72 \mathrm{~h}$ of $\mathrm{LiCl}$ treatment led to decreased cell growth when cells were transfected with control siRNA but had no effect on $\beta$-catenin knock down cells (b) $24 \mathrm{~h}$ and $48 \mathrm{~h}$ post transfection a $>70 \%$ knock-down of $\beta$-catenin was examined by qRT-PCR.

Additional file 6: Figure S6. Treatment of nude mice with $\mathrm{NaCl}$ or $\mathrm{LiCl}$ did not change vessel density, amount of cleaved caspase positive cells or aSMA positive cells. Immunohistochemistry with (a) anti-cleaved Caspase 3 (Cell Signaling) on paraffin-embedded sections ( $3 \mu \mathrm{m}$ ) of flank tumors or (b) anti-cleaved Caspase 3 (Cell signaling) on paraffin-embedded cell pellets and (d) anti-CD31 antibody (Clone SZ31, Dianova, Hamburg, Germany) was performed using the automated Ventana Discovery XT staining system (Ventana, Tucson, Arizona USA) and standard protocols. Slides were examined with the Axiophot light Microscope (Zeiss, Germany, Achroplan 0.65) and analyzed with the Stereo Investigator Software 4.34 (MicroBright Field. Inc Europe, Magdeburg, Germany). Pictures were taken at a light microscope (Nikon Eclipse 80i; Nikon, Japan; 10x/40x; NA 0.5). Bars represent mean \pm s.d. for $\mathrm{NaCl}$ (grey) or LiCl (black) treatment (CD31: $n=6(\mathrm{NaCl}), n=6$ (LiCl); cleaved Caspase 3: $n=6$ ( $\mathrm{NaCl}), n=4$ (LiCl); cleaved Caspase 3 cell pellet: $n=1$ ( $/ \mathrm{NaCl}$ and LiCl)). Scale bar represent $200 \mu \mathrm{m}$ (left) and $50 \mu \mathrm{m}$ (right). (c) Immunofluorescence staining with anti-a-smooth muscle actin-Cy3 antibody was performed on paraffin-embedded sections $(3 \mu \mathrm{M})$. Staining intensity was measured with ImageJ 1.47v software and normalized to tumor size.

Additional file 7: Figure S7. Histo-pathological characterization of subcutaneous $\mathrm{Ptch}^{+-}$tumors. Paraffin-embedded sections $(3 \mu \mathrm{m})$ of flank tumors were stained with hematoxylin and eosin. Immunohistochemistry with anti-GFAP antibody (DakoCytomation) and reticulin (special stain Ventana) on paraffin-embedded sections $(3 \mu \mathrm{m})$ of flank tumors was performed using the automated Ventana Discovery XT staining system (Ventana, Tucson, Arizona USA) and standard protocols. Stainings were visualized by Olympus BX50 light microscope 10× (NA 0.30). Scale bar represent $200 \mu \mathrm{m}$

\section{Abbreviations}

Shh: Sonic hedgehog; MB: Medulloblastoma; Ptch ${ }^{+/}$: Patched heterozygous; Hh: Hedgehog; Ptch1: Patched1; 6BIO: (2'Z,3'E)-6-Bromoindirubin-3'-oxime; MEFs: Mouse embryonic fibroblasts; PLA: Proximity ligation assay; ShhCM: Sonic hedgehog-conditioned medium; pHH3: Phospho-Histone H3; LiCl: Lithium-chloride; NaCl: Sodium-chloride.

\section{Competing interests}

The authors declare that they have no competing interests.

\section{Authors' contributions}

JZ conducted in vivo experiments and wrote the manuscript; JZ and FTS conceived and performed experiments; analyzed and interpreted data; PNH analyzed and provided neuropathology expertise; ST took care of animals; performed in vivo experiments; NZ analyzed and interpreted data; RT provided cells; interpreted data; KHP provided neuropathology expertise, SL designed and supervised the study; wrote the manuscript; performed in vivo experiments. All authors read and approved the final manuscript.

\section{Acknowledgements}

We thank Maika Dunst and Tatjana Starzetz for sectioning paraffin embedded samples and provided help with the Ventana Staining Device. We thank Jadranka Macas for FACS analyzing of cell cycle kinetics. We thank Kavi Devraj for statistical analysis. We thank Dr. Csaba Finta for providing the HEK293 ShhCM. We thank Manuela Hugle and Professor Simone Fulda for providing us the pHH3 antibody and a detailed staining protocol, and Raj Cumar Vutukuri and PD Dr. Waltraud Pfeilschifter for scanning aSMA stained slides. This study has been supported by the Deutsche Forschungs Gemeinschaft (SFB/TR23 B7 "Vascular Differentiation and Remodeling" to S.L.), the LOEWE Initiative Hessen, (Onkogene Signaltransduktion Frankfurt, OSF; III L 4-518/ 55.004, 2009 to KHP and S.L.), the Excellence Cluster Cardio-Pulmonary System (ECCPS, to KHP and S.L.) and by EU Health FP7 JUSTBRAIN to KHP and S.L.; F.T.S. was supported by the Wenner-Gren Foundation (Stockholm, Sweden).

\section{Author details}

'Institute of Neurology (Edinger-Institute), Johann Wolfgang Goethe-University Frankfurt, Medical School, Heinrich-Hoffmann-Straße 7, 60528 Frankfurt, Germany. ${ }^{2}$ Center for Biosciences and Department of
Biosciences and Nutrition, Karolinska Institutet, Novum, Huddinge, Sweden. ${ }^{3}$ German Cancer Consortium (DKTK) and German Cancer Research Center (DKFZ), Heidelberg, Germany. ${ }^{4}$ Current address: Department of Neuropathology, Institute of Pathology and Pathological Anatomy, Technical University Munich, Trogerstrasse 18, 81675 Munich, Germany.

Received: 24 July 2014 Accepted: 12 January 2015 Published online: 03 February 2015

\section{References}

1. Liu H, Fergusson MM, Castilho RM, Liu J, Cao L, Chen J, et al. Augmented Wnt signaling in a mammalian model of accelerated aging. Science. 2007;317:803-6.

2. Taylor MD, Northcott PA, Korshunov A, Remke M, Cho Y-J, Clifford SC, et al. Molecular subgroups of medulloblastoma: the current consensus. Acta Neuropathol. 2012;123:465-72.

3. Liu H, MacCallum D, Edwards C, Gaffield W, Mistretta C. Sonic hedgehog exerts distinct, stage-specific effects on tongue and taste papilla development. Dev Biol. 2004;276:280-300.

4. Schneider FT, Schänzer A, Czupalla CJ, Thom S, Engels K, Schmidt MHH, et al. Sonic hedgehog acts as a negative regulator of $\{$ beta\}-catenin signaling in the adult tongue epithelium. Am J Pathol. 2010;177:404-14.

5. Pöschl J, Bartels M, Ohli J, Bianchi E, Kuteykin-Teplyakov K, Grammel D, et al. Wnt/ $\beta$-catenin signaling inhibits the Shh pathway and impairs tumor growth in Shh-dependent medulloblastoma. Acta Neuropathol. 2014;127(4):605-7.

6. Anne SL, Govek E-E, Ayrault O, Kim JH, Zhu X, Murphy DA, et al. WNT3 inhibits cerebellar granule neuron progenitor proliferation and medulloblastoma formation via MAPK activation. PLoS One. 2013;8:e81769.

7. Northcott PA, Jones DTW, Kool M, Robinson GW, Gilbertson RJ, Cho Y-J, et al. Medulloblastomics: the end of the beginning. Nat Rev Cancer. 2012;12:818-34.

8. Goodrich L, Milenkovic L, Higgins K, Scott M. Altered neural cell fates and medulloblastoma in mouse patched mutants. Science. 1997;277:1109-13.

9. Mueller S, Chang S. Pediatric brain tumors: current treatment strategies and future therapeutic approaches. Neurotherapeutics. 2009;6:570-86.

10. Clevers H. Wnt/beta-catenin signaling in development and disease. Cell. 2006;127:469-80

11. Alvarez-Medina R, Cayuso J, Okubo T, Takada S, Martí E. Wnt canonical pathway restricts graded Shh/Gli patterning activity through the regulation of Gli3 expression. Development. 2008;135:237-47.

12. Ingham PW. Hedgehog signalling. Curr Biol. 2008;18:R238-41.

13. Yin Y, Kizer NT, Thaker PH, Chiappinelli KB, Trinkaus KM, Goodfellow PJ, et al. Glycogen synthase kinase $3 \beta$ inhibition as a therapeutic approach in the treatment of endometrial cancer. Int J Mol Sci. 2013;14:16617-37.

14. Nowicki MO, Dmitrieva N, Stein AM, Cutter JL, Godlewski J, Saeki Y, et al. Lithium inhibits invasion of glioma cells; possible involvement of glycogen synthase kinase-3. Neuro Oncol. 2008;10:690-9.

15. Peng Z, Ji Z, Mei F, Lu M, Ou Y, Cheng X. Lithium inhibits tumorigenic potential of PDA cells through targeting hedgehog-GLI signaling pathway. PLoS One. 2013;8:e61457.

16. Meijer L, Flajolet $M$, Greengard P. Pharmacological inhibitors of glycogen synthase kinase 3. Trends Pharmacol Sci. 2004;25:471-80.

17. Rhee J, Ryu JH, Kim JH, Chun CH, Chun JS. a-Catenin inhibits $\beta$-catenin-Tcf/ Lef transcriptional activity and collagen type II expression in articular chondrocytes through formation of a Gli3R/a-catenin/ $\beta$-catenin ternary complex. J Biol Chem. 2012;287(15):11751-60.

18. Liao X, Siu MK, Au CW, Chan QK, Chan HY, Wong ES, et al. Aberrant activation of hedgehog signaling pathway contributes to endometrial carcinogenesis through. Mod Pathol. 2009;22:839-47.

19. Kim J-H, Shin HS, Lee SH, Lee I, Lee YS, Park JC, et al. Contrasting activity of Hedgehog and Wnt pathways according to gastric cancer cell differentiation: relevance of crosstalk mechanisms. Cancer Sci. 2010;101:328-35.

20. Vleminckx K, Kemler R, Hecht A. The C-terminal transactivation domain of beta-catenin is necessary and sufficient for signaling by the LEF-1/beta-catenin complex in Xenopus laevis. Mech Dev. 1999;81:65-74.

21. Veeman MT, Slusarski DC, Kaykas A, Louie SH, Moon RT. Zebrafish prickle, a modulator of noncanonical Wnt/Fz signaling, regulates gastrulation movements. Curr Biol. 2003;13:680-5.

22. Schou M. Forty years of lithium treatment. Arch Gen Psychiatry. 1997;54:9-13. discussion 14-5. 
23. Walter CJ, Bell LTO, Parsons SR, Jackson C, Borley NR, Wheeler JMD. Prevalence and significance of anaemia in patients receiving long-course neoadjuvant chemoradiotherapy for rectal carcinoma. Colorectal Dis. 2013;15:52-6.

24. Reis M, Czupalla CJ, Ziegler N, Devraj K, Zinke J, Seidel S, et al. Endothelial Wnt/B-catenin signaling inhibits glioma angiogenesis and normalizes tumor blood vessels by inducing PDGF-B expression. J Exp Med. 2012;209:1611-27.

25. Reis M, Liebner S. Wnt signaling in the vasculature. Exp Cell Res. 2013;319:1317-23.

26. Kool M, Jones DTW, Jäger N, Northcott PA, Pugh TJ, Hovestadt V, et al. Genome sequencing of SHH medulloblastoma predicts genotype-related response to smoothened inhibition. Cancer Cell. 2014;25:393-405.

\section{Submit your next manuscript to BioMed Central and take full advantage of:}

- Convenient online submission

- Thorough peer review

- No space constraints or color figure charges

- Immediate publication on acceptance

- Inclusion in PubMed, CAS, Scopus and Google Scholar

- Research which is freely available for redistribution 